\title{
Patterns of Cancer Care Costs in a Country With Detailed Individual Data
}

\author{
Tony Blakely, PhD, * June Atkinson, NZC Statistics, * Giorgi Kvizhinadze, PhD,* \\ Nick Wilson, MPH,* Anna Davies, MSc,* and Philip Clarke, PhD†
}

Objective: To determine health system expenditure on cancers by time since diagnosis using data for an entire country.

Methods: New Zealand cancer registry data was linked to hospitalization, pharmaceutical, outpatient, general practice, laboratory, and other datasets, with costs ascribed to each event occurring in 2006-2011. "Excess" cancer costs were estimated by subtracting "expected costs" for citizens without cancer from the "total cost" for cancer patients (\$2011 inflation-adjusted). Gamma regressions were used to estimate costs per person-month.

Results: For first adult cancer diagnosed that the excess cost per person was between US\$3400 and US\$4300 in the first month postdiagnosis (varied by sex and age), fell to US\$50-US\$150 per month at 2 or more years postdiagnosis (excluding those within a year of death), but increased again if dying from their cancer (US\$3800-US\$8300 in the last month of life). Such patterns varied by cancer, for example, in the first month postdiagnosis for 65 year olds it varied 20 -fold from US $\$ 800$ for prostate to US $\$ 15,900$ for brain cancer. Per diagnosed case, total excess costs varied from US\$8000 (melanoma) to US\$98,000 (bone and connective tissue). Excess cancer costs made up $6.5 \%$ of total Vote:Health expenditure in 2010-2011, with colorectal (14.7\%), breast (14.4\%) being the top 2 contributors, and prostate, non-Hodgkin lymphoma, leukemia, and lung each contributing about $6 \%$.

Conclusions: Costs vary substantially by time since diagnosis and cancer type. The results and regression equations reported in this paper can be used in modeling requiring cancer costs by time since diagnosis and proximity to death.

From the *Department of Public Health, University of Otago, Wellington, New Zealand; and †Centre for Health Policy, Programs and Economics School of Population Health, The University of Melbourne, Parkville, VIC, Australia.

Supported by Health Research Council of New Zealand (10/248), and in kind by the Ministry of Health.

The authors declare no conflict of interest.

Reprints: Tony Blakely, $\mathrm{PhD}$, Department of Public Health, University of Otago, Wellington, P.O. Box 7343, Wellington South 6242, New Zealand. E-mail: tony.blakely@otago.ac.nz.

Supplemental Digital Content is available for this article. Direct URL citations appear in the printed text and are provided in the HTML and PDF versions of this article on the journal's Website, www.lww-medical care.com.

Copyright (C) 2015 Wolters Kluwer Health, Inc. All rights reserved. This is an open-access article distributed under the terms of the Creative Commons Attribution-NonCommercial-NoDerivatives 3.0 License, where it is permissible to download and share the work provided it is properly cited. The work cannot be changed in any way or used commercially.

ISSN: 0025-7079/15/5304-0302
Key Words: cancer, health system, costs

(Med Care 2015;53: 302-309)

$\mathrm{N}$ oncommunicable diseases are now the major contributor to the burden of disease in high-income countries and most low-income and middle-income countries. ${ }^{1}$ In many countries, cardiovascular disease incidence is falling and cancer is emerging as an important driver of disease burden and health care costs. In recent years we have witnessed the development of life extending drugs for many types of cancer which typically costs upward of US\$60,000 per life-year gained. ${ }^{2,3}$

While the frameworks for evaluating cancer therapies vary across countries, ${ }^{4}$ a common consideration in health technology assessment is the magnitude of any cost offsets in terms of the scope for the new cancer drugs to reduce the current cost of health care for these patients. ${ }^{5}$ Thus an intervention that prevents cancer can "save" future health care costs, and the timing of these averted costs is also an element of any evaluation. An additional timing consideration is the costs of cancer patients in the final few months of life; as a treatment that prolongs life is likely to shift these costs further into the future. For these reasons, costing breakdowns by time leading up to diagnosis, postdiagnosis, and in months before dying from cancer can be used to inform economic evaluations. $^{6}$

Economic evaluations of interventions to treat and prevent cancer use varying approaches to health system costs across the cancer pathway, making such evaluations noncomparable. ${ }^{7}$ A superior approach would be to development cancer reference costs that employ a common methodology as well as disaggregating these costs over time to understand variations of the course of the disease (eg, costs at time of diagnosis vs. cost in the last few months of life). Further cancer-specific costs (by sex and age) are a valuable source for planning as the funding requirements for health care services require understanding where costs are incurred and what are likely to be the future cost drivers.

Surprisingly, there are relatively few studies that quantify excess cost of health care for patients diagnosed with a wide range of cancers (ie, where excess costs are the additional cost of cancer care for a patient above the expected cost of health care). One exception is by Mariotto et $\mathrm{al},{ }^{8}$ who estimate costs of cancer care for United States up to 2020. It is based on health care costs of Medicare 
beneficiaries (ie, those aged over $65 \mathrm{y}$ ) during the initial period after diagnosis, the final year of life, and the period between those times.

Given this background, we aimed to cost multiple cancers by time preceding and after diagnosis for New Zealand (population 4.4 million). This is a country with detailed individual-level costing data across the entire public health care system that can be aggregated at the national level. (About $83 \%$ of health system expenditure in New Zealand is public expenditure.) In particular, the objectives of this paper are: (1) to estimate the expenditure on cancer patients, by cancer site, for time leading up to and following diagnosis, and separately if the cancer patient is in the last 12 months of life; and (2) to estimate the total public health system costs due to cancer using an excess costing method and to make comparisons with an alternative "attribution" costing method; and (3) to look at the degree to which survival rates are related to costs across different types of cancers.

\section{METHODS}

Patients included in the analyses were all usually resident New Zealand cancer patients, with a "prevalent" cancer at any point in the July 2006 to June 2011 period. It was possible to include all cancer patients due to the existence on nationally linked public sector health datasets, covering the entire country, while patients continue to be resident in New Zealand.

\section{Linked Administrative Health Care Datasets With Costs Per Event}

The New Zealand health system has a unique individual identifier of high quality since about 1990 (the National Health Index number). The following datasets were linked using this identifier to create a record for each New Zealander of publicly funded (Vote:Health) events occurring between July 2006 and June 2011: hospitalizations and inpatient procedures ${ }^{9}$ (including inpatient laboratory tests and pharmaceuticals); community laboratory tests; nonadmitted patient events (eg, outpatients); community pharmaceuticals dispensed (including patient contribution); general practice consultations (both that calculated based on a capitation funding formula, and fee for service when not an enrolled patient in a capitated practice). Each linked event was then assigned a unit cost (eg, hospitalizations and inpatient procedures using Ministry of Health cost weights per event ${ }^{9}$ ). (The following event datasets were not included: injuries (ie, that funded through Vote: Accident Compensation Corporation), disability support services (eg, rest homes), maternity care outside of hospital and mental health). By way of example, for the 2010-2011 financial year, $\$ 6.73$ billion of Vote:Health was assigned to "events" [50\% of $\$ 13.57$ billion total, the unassigned remainder being due to excluded events (eg, mental health and maternity care) and costs not attributable to an event (eg, public health, administration)]. Goods and services tax was excluded. All costs were inflation-adjusted to 2011 dollars, and are given in NZ\$ unless stated otherwise.

\section{Cancer Patients' Costs and Person-Time Allocation}

New Zealand has a national cancer registry with compulsory registration (covering all cancers other than for nonmalignant skin cancers). We have previously determined statistical cure times by cancer ${ }^{10}$ (ie, the number of years postdiagnosis at which cancer excess mortality is negligible), ranging from 3 years (eg, testicular) to 20 years (eg, breast, prostate, myeloma, and non-Hodgkin lymphoma; Table 1); prevalent cases were those within the statistical cure time since their diagnosis.

Some people contribute person-time and costs to 2 cancers simultaneously if they were prevalent cases for 2 or more cancers at any given time. For the "first diagnosed adult cancer" group across all sites we only included person-time for the first cancer diagnosed that had a cure time (3-20y postdiagnosis) encroaching into the 2006-2011 period.

Person-time was allocated by phase of the cancer management pathway: $6-11,1-5,<1$ month prediagnosis and $<1,1-5,6-11,12-23$, and $24+$ month postdiagnosis (broadly consistent with previous studies ${ }^{13}$ ); plus 3 categories for patients within $6-11,1-5,<1$ months of death from the given cancer (person-time was prioritized to time preceding death). Costs were allocated evenly across the duration of any hospital event (eg, an "end-date" cost for someone hospitalized for 3 months preceding their death from cancer had $1 / 3$ of this cost assigned to "the last month of life" category, and $2 / 3$ to the " $1-5$ months preceding death" category).

\section{Excess Costs Due to Cancer}

We did not attempt to classify what was and was not a specific "cancer-related" cost. Rather, we used the established "excess" or "net" cost approach, ${ }^{8,14-16}$ whereby we calculated the expected health system cost of a New Zealand citizen by sex and age group without the cancer diagnosis, ${ }^{17}$ then subtracted this from the observed total costs. This excess cost is assumed to equate to the costs due to the cancer diagnosis and management, a similar approach to that used in relative survival and excess mortality rate modeling with cancer registry data. ${ }^{18,19}$ If the total observed cost for a cancer strata was actually less than the expected cost, we forced the excess cost to be negligible (ie, $\$ 1$ per month).

We converted some of the NZ\$ values to US\$ for comparative purposes, using the OECD 2011 benchmark purchasing power parity of 1.486 .

\section{Analyses}

The total costs across the population for 2010-2011 was calculated by simple summing of excess cancer costs.

For each cancer site we ran log-link gamma regression models (Proc Genmod SAS 9.4) on 2006-2011 data disaggregated to the level of all possible cross-classified strata of sex by 5-year age group by phase (ie, person-time) of the cancer management pathway by financial year. For each possible strata, the number of uniquely contributing individuals, person-time (mo) and sum of all health systems costs were calculated-and thence "excess costs" by 
TABLE 1. Descriptive Data and Costs by Cancer Site

\begin{tabular}{|c|c|c|c|c|c|c|c|c|}
\hline \multirow[b]{2}{*}{ Cancer } & \multirow[b]{2}{*}{$\begin{array}{c}\text { ICD } 10 \\
\text { Code }\end{array}$} & \multirow[b]{2}{*}{$\begin{array}{l}\text { Statistical } \\
\text { Cure Time } \\
\text { (y) }\end{array}$} & \multirow[b]{2}{*}{$\begin{array}{l}\text { Person- } \\
\text { Years } \\
\text { 2006-2011 }\end{array}$} & \multirow[b]{2}{*}{$\begin{array}{c}\text { Incidence } \\
\text { Rate } \\
\text { 2010 }^{*} \dagger\end{array}$} & \multirow[b]{2}{*}{$\begin{array}{c}5 \mathrm{Y} \\
\text { Relative } \\
\text { Survival }^{*}\end{array}$} & \multicolumn{3}{|c|}{ Excess Costs in 2010-2011 (in NZ\$2011) } \\
\hline & & & & & & $\begin{array}{l}\text { Millions for all } \\
\text { Prevalent } \\
\text { Cases }\end{array}$ & $\begin{array}{c}\text { \% of "Total" } \\
\text { for Prevalent } \\
\text { Cases }\end{array}$ & $\begin{array}{c}\text { Approximate Cos } \\
\text { Per Diagnosed } \\
\text { Case }\end{array}$ \\
\hline Lip, oral cavity, pharynx & $\mathrm{C} 00-14$ & 10 & 123,461 & 7.1 & & $\$ 21.6$ & 2.5 & $\$ 52,000$ \\
\hline Esophagus & $\mathrm{C} 15$ & 6 & 29,826 & 4.4 & 0.117 & $\$ 10.6$ & 1.2 & $\$ 36,000$ \\
\hline Stomach & $\mathrm{C} 16$ & 6 & 53,687 & 5.9 & 0.225 & $\$ 16.8$ & 1.9 & $\$ 46,000$ \\
\hline Colorectal and anus & C18-21 & 8 & 838,162 & 45.0 & 0.602 & $\$ 129.7$ & 14.7 & $\$ 43,000$ \\
\hline Rectum & $\mathrm{C} 20$ & 8 & 217,524 & 11.3 & & $\$ 44.7$ & 5.1 & $\$ 62,000$ \\
\hline Anus and anal canal & $\mathrm{C} 21$ & 8 & 16,150 & 1.0 & & $\$ 3.3$ & 0.4 & $\$ 54,000$ \\
\hline Liver & $\mathrm{C} 22$ & 7 & 27,789 & 4.1 & 0.142 & $\$ 9.1$ & 1.0 & $\$ 35,000$ \\
\hline Gallbladder & C23-24 & 7 & 13,560 & 1.7 & & $\$ 4.2$ & 0.5 & $\$ 35,000$ \\
\hline Pancreas & $\mathrm{C} 25$ & 5 & 32,339 & 7.3 & 0.070 & $\$ 11.5$ & 1.3 & $\$ 23,000$ \\
\hline Larynx & C32 & 10 & 30,260 & 1.2 & & $\$ 5.7$ & 0.6 & $\$ 76,000$ \\
\hline Lung, trachea, bronchus & C33-34 & 6 & 188,974 & 29.5 & 0.111 & $\$ 55.9$ & 6.4 & $\$ 29,000$ \\
\hline Uterus & C5455 & 6 & 121,732 & 8.4 & 0.754 & $\$ 12.6$ & 1.4 & $\$ 25,000$ \\
\hline Ovary & C56 & 10 & 85,391 & 5.0 & 0.426 & $\$ 13.3$ & 1.5 & $\$ 44,000$ \\
\hline Prostate & C61 & 20 & $1,584,361$ & 46.9 & 0.862 & $\$ 48.6$ & 5.5 & $\$ 16,000$ \\
\hline Testes $^{\S}$ & C62 & 3 & 25,986 & 4.0 & 0.962 & $\$ 3.4$ & 0.4 & $\$ 21,000$ \\
\hline Kidney, urinary & $\begin{array}{c}\text { C64-66, } \\
\text { C68 }\end{array}$ & 10 & 160,861 & 9.5 & 0.576 & $\$ 23.7$ & 2.7 & $\$ 40,000$ \\
\hline Bladder & C67 & 10 & 157,155 & 5.4 & 0.669 & $\$ 14.3$ & 1.6 & $\$ 37,000$ \\
\hline Brain & C71 & $5 / 10^{\|}$ & 36,602 & 5.8 & 0.190 & $\$ 18.9$ & 2.2 & $\$ 60,000$ \\
\hline Thyroid $^{\S}$ & $\mathrm{C} 73$ & 5 & 31,039 & 5.1 & 0.905 & $\$ 2.4$ & 0.3 & $\$ 18,000$ \\
\hline Hodgkin lymphoma & $\mathrm{C} 81$ & 10 & 44,326 & 2.2 & 0.824 & $\$ 6.6$ & 0.8 & $\$ 65,000$ \\
\hline Non-Hodgkin lymphoma & $\begin{array}{c}\mathrm{C} 82-85, \\
\text { C96 }\end{array}$ & 20 & 302,601 & 12.8 & 0.564 & $\$ 56.4$ & 6.4 & $\$ 72,000$ \\
\hline Myeloma & $\mathrm{C} 90$ & 20 & 76,257 & 4.7 & 0.352 & $\$ 24.8$ & 2.8 & $\$ 80,000$ \\
\hline Leukemia & C91-95 & 10 & 212,690 & 10.2 & 0.510 & $\$ 55.9$ & 6.3 & $\$ 95,000$ \\
\hline
\end{tabular}

*Age standardized (to world population) incidence rate per 100,000 population.

Source: Ministry of Health. ${ }^{11}$

${ }^{\star}$ Source: Ministry of Health. ${ }^{12}$

${ }^{\S}$ Testes and thyroid cancer person-time and cost is only for ages $<50$ years.

$\| 5$ years for cases $<55$ years; 10 years for cases $\geq 55$ years

ICD 10 codes: C17, C26, C30-C31, C37-C39, C44-C49, C51-C52, C57-C58, C60, C63, C69-C70, C72, C74, C76-C80, C88.

${ }^{\#}<25$ years at diagnosis, and also included in the cancer sites above.

* * Substantially $<100 \%$ due to both exclusion of children, but also exclusion of people with diagnosed cancer in 2010-2011 who have previous cancer diagnosis within last 3 years (eg, testes, thyroid) to 20 years (eg, myeloma, leukemia).

subtracting off average citizen linked costs expected for the given sex, age, and person-time. The dependent variable in the gamma regression was excess cost, the off-set was person-time (mo), the forced (ie, had to stay in the model) independent variables were sex, year (centered on 2011), age (as a continuous variable using the value for the midpoint of each 5-y age category, centered on $62.5 \mathrm{y}$, then divided by 10 ) and the categorical phase variable (ie, time prediagnosis and postdiagnosis, or time before death). "Optional" variables included age-squared, age-cubed, and an age (linear only, not quadratic or cubic) by phase interaction.

For each cancer, the following strategy was used to select the "best" regression equation. First, outliers were identified in initial regressions and omitted using standardized Pearson residual $(<-4$ or $>5)$, likelihood residuals $(<-7$ or $>7)$, leverage statistic $(>0.1)$, Cook $D(>0.1)$. Second, "optional" variables were removed with a backwards selection process on the basis of Akaike and Bayesian information criteria, deviance statistics, and $P$-values (exact details available from authors on request).

These regression equations were then used to estimate "smoothed" excess costs due to cancer, per person-month, by phase of the cancer management pathway in 2011.

Finally, we examined the association between cost and relative cancer survival, using 5-year relative survivals published elsewhere. ${ }^{12}$ 


\section{RESULTS}

Table 1 shows descriptive statistics by cancer site (and distribution of by sex and age in Supplementary Table 1, Supplemental Digital Content 1, http://links.lww.com/MLR/ A879).

\section{Total Cost of Cancer in 2010-2011}

Also shown in Table 1 are excess costs by cancer site for 2010-2011. Summed across all prevalent cancer patients regardless of phase of the cancer management pathway, the cost was NZ\$880 million (US\$592 million), equivalent to $6.5 \%$ of Vote:Health [ $\$ 13.57$ billion in 2011-2012) or $13.0 \%$ of expenditure captured by HealthTracker (see the Methods section)]. Colorectal cancer contributed the most to cancer excess costs (14.7\%), followed by breast (14.4\%). Prostate, lung, non-Hodgkin lymphoma, and leukemia each contributed $5 \%-7 \%$.

\section{Cost of Cancer by Phase of Cancer Management Pathway}

Costs varied markedly by phase of the cancer pathway (Table 2). For the reference person [male, age 60-64 (ie, $62.5 \mathrm{y}$ ), year 2011], predicted costs for the first adult cancer diagnosis were maximal (compared with 24 or more months postdiagnosis and not within a year of death from their cancer) for people within 1 month of death from cancer [rate ratio (RR) 65.7]. The next largest cost per month was in the first month postdiagnosis (RR 41.1), followed by $1-5$ months preceding death (RR 28.8) and 1-5 months postdiagnosis (RR 18.2).

Figure 1 shows the excess health system cost for the first adult cancer, by time preceding and postdiagnosis, for males and females aged 45,65 , and 85 years. (The values are those from the gamma regression coefficients shown in Table 2.) The excess cost per person was between $\$ 5030$ and $\$ 6440$ in the first month postdiagnosis (US\$3400US\$4300), fell to $\$ 69-\$ 221$ per month at 2 or more years postdiagnosis (US\$50-US\$150; excluding those within a year of death), but increased again to $\$ 5660-\$ 12,300$ in the last month of life if dying from their cancer (US\$3800US\$8300; notably higher if younger at death).

This pattern varied by cancer; in the first month postdiagnosis for 65 year olds, the excess cost varied 20 -fold from US $\$ 1190$ for prostate to $\$ 23,700$ for brain cancer (US\$800-US\$15,900; Fig. 2). (The gamma regression equations used to generate the cancer-specific excess costs are shown in Supplementary Table 2, Supplemental Digital Content 2, http://links.lww.com/MLR/A880 accompanied by instructions for readers to generate cost by phase for any sex by age combination.) Other lower cost cancers in the first month postdiagnosis include those for breast, cervix, uterus, and prostate. In the 1-5 months postdiagnosis, costs tended to fall, but the relative ranking of cancers was similar. Costs varied only about 3- to 4-fold in the last month of life if dying from cancer (Supplementary Figure 1, Supplemental Digital Content 3, http://links.lww.com/MLR/A881).

For nearly all first adult cancer by sex by age groupings shown in Figure 1, the cancer costs were greater for 45 year olds relative to 65 year olds and especially to 85 year olds.
Figure 3 shows a plot of the cost per diagnosed cancer (ie, that in the last column of Table 1) and the relative survival (also in Table 1). A quadratic regression has been fitted to illustrate the inverted U-shaped relationship between cost of each cancer and the relative survival. Costs per case increase up to a relative survival of $0.2-0.3$, are roughly similar to a relative survival of about 0.6 , then tend to decrease with improving survival of the cancer.

\section{DISCUSSION}

We estimated public health expenditure on cancer using internationally accepted excess costing methodology, ${ }^{8,14-16}$ whereby the quantum of health system spending on cancer patients over and above that expected for comparable citizens is estimated. In 2010-2011 \$880 million of health costs (US\$592 million) was due to cancer, or about $6.5 \%$ of Vote:Health. The largest expenditure per month was in the last month of life (if dying from the cancer) and the first month postdiagnosis. However, patterns of expenditure by phase of the cancer management pathway differed by cancer site. For example, melanoma and prostate cancers had relative low expenditure postdiagnosis compared with other cancers. The variation in per month expenditure on cancers was most marked immediately postdiagnosis, but before death there was less variation in the cost per month.

We also found that cancers with a 5-year relative survival ranging from about 0.25 to 0.60 tended to have the highest cancer costs per diagnosed case (Fig. 3). This pattern is consistent with: poor prognosis cancer patients not being alive long enough to generate high excess costs; good prognosis cancers responding to initial treatment more fully; and average prognosis cancers consuming more resource due to recurrences, and available treatments that make a useful impact on absolute survival chances (as opposed to small marginal gains of treatment when survival is close to $0 \%$ or $100 \%$ ). The latter is suggestive of relatively rational decision making (from a health economics perspective) by clinicians and those developing guidelines for cancer management. So is the greater investment in cancer management for 45 year olds relative to older age groups (when considering the potential in life-years gained from treatment successes).

In the United States, Mariotto et $\mathrm{al}^{8}$ have estimated costs of cancer (using a similar excess method to ours), with costs in the first year of diagnosis ranging from US $\$ 6000$ (2010 US\$; melanoma) to US\$135,000 (brain cancer). The ranking of cancers by such costs were similar to our findings for New Zealand, but the quantum was much greater in the United States where health expenditure per capita is very much higher. Using 65-year-old males in Figure 1, the excess cost in the first year of diagnosis for first adult cancer was NZ $\$ 22,358(\$ 5650+5 \times \$ 2420+6 \times \$ 768$; US $\$ 15,000)$, considerably less than the average across cancers in the United States. However, the patterns of cost by time since diagnosis and predeath are similar. ${ }^{8,20,21}$

The linked New Zealand data (so-called "HealthTracker") has been used to estimate the "price of cancer" previously, by the Ministry of Health. ${ }^{22}$ They estimated a total cost of NZ\$511 million in 2009 (\$526 million in 2011 inflation- 
TABLE 2. Gamma Regression Coefficients and RR for First Adult Cancer

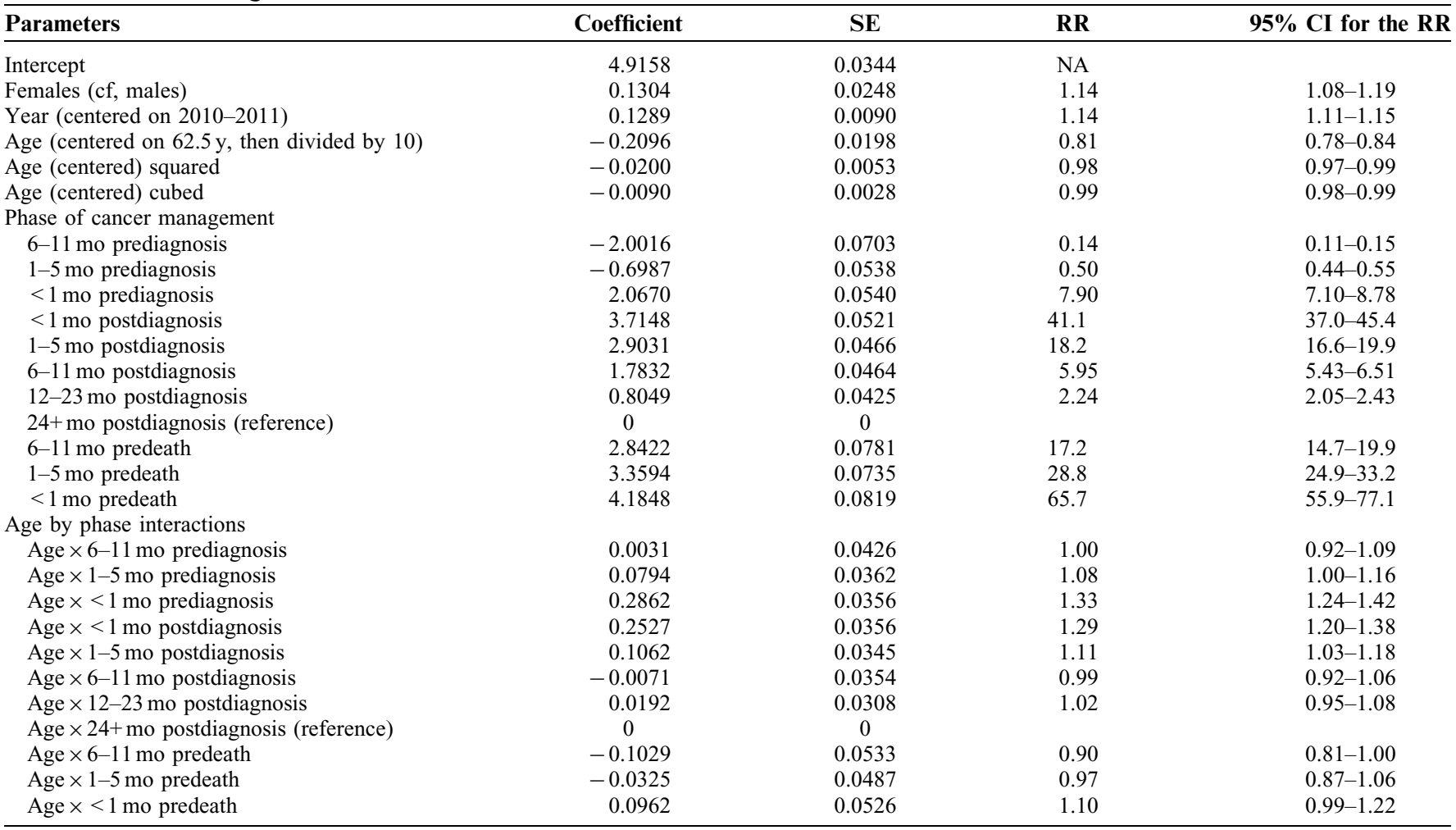

Regressions were weighted by the number of cases in each stratum.

$\mathrm{CI}$ indicates confidence interval; RR, rate ratio.

adjusted NZ\$), compared with our total cost of $\$ 880.4$ million (2011 NZ\$). The Ministry estimate was for first cancer using a 5-year cure time or look back period uniformly for all cancers, meaning costs of a second cancer within 5 years in the same person are excluded. We also calculated a "first adult cancer" cost (\$628.6 million), which added to childhood cancer ( $\$ 32.2$ million) gives $\$ 660.9$ million-still 26\% larger than the Ministry method. But we used a differing (but usually larger) cure time and look back period to define "first case," from 3 years (eg, testicular cancer) to 20 years (eg, breast, prostate, myeloma, and nonHodgkin lymphoma; Table 1), depending on how long postdiagnosis excess mortality from the cancer diagnosis is occurring-so-called statistical cure time. ${ }^{10}$ Our method will therefore include the excess costs of treating cancer relapse 5 or more years postdiagnosis, and (in particular) the costs of people dying of their cancer 5 or more years postdiagnosis, driving up costs relative to the Ministry method. Conversely, we did not include someone with a "new" cancer if they had (say) a breast or prostate cancer in the last 20 years, driving down cost relative to the Ministry method for "first cancer." That all said, it is clear that our cost estimates are greater than the Ministry of Health, even allowing for these cure time differences. A substantive reason for this is probably that the Ministry method only counted those health inpatient and outpatient events that were "obviously" coded as being due to cancer, and should thus be treated as an overly con- servative or lower bound estimate. For example, if someone's cancer diagnosis caused their previously well-controlled angina to become difficult to manage with attendant admissions to hospital, then that would not have been captured by the Ministry method but would have been captured with our excess cost method.

An important motivation behind this analysis has been to develop a set of costs for all major cancers using the same costing methodology that can be used to support economic evaluations. The development of such reference costs is particularly timely given the significant number of new therapies to treat or prevent cancer that need to be evaluated for decisions around adoption and reimbursement. ${ }^{2}$ As our analysis has shown that some of the highest average costs are in the last few months of life, it means that if treatments prolong survival, the cost profiles of these cancers will change in the future. It is also likely that increased survival rates across all cancers will produce a dividend in that it pushes the expensive last few months of life further into the future (a benefit when costs are subjected to discount rates). To facilitate use of the costs reported in this paper, we have provided instructions and examples of how to estimate the cost associated with different types of cancer based on the gamma regression equations in the Supplementary material (Supplemental Digital Content 2, http://links.lww.com/MLR/A880).

The HealthTracker data used in these analyses is, by international standards, an uncommonly rich dataset. But it is 


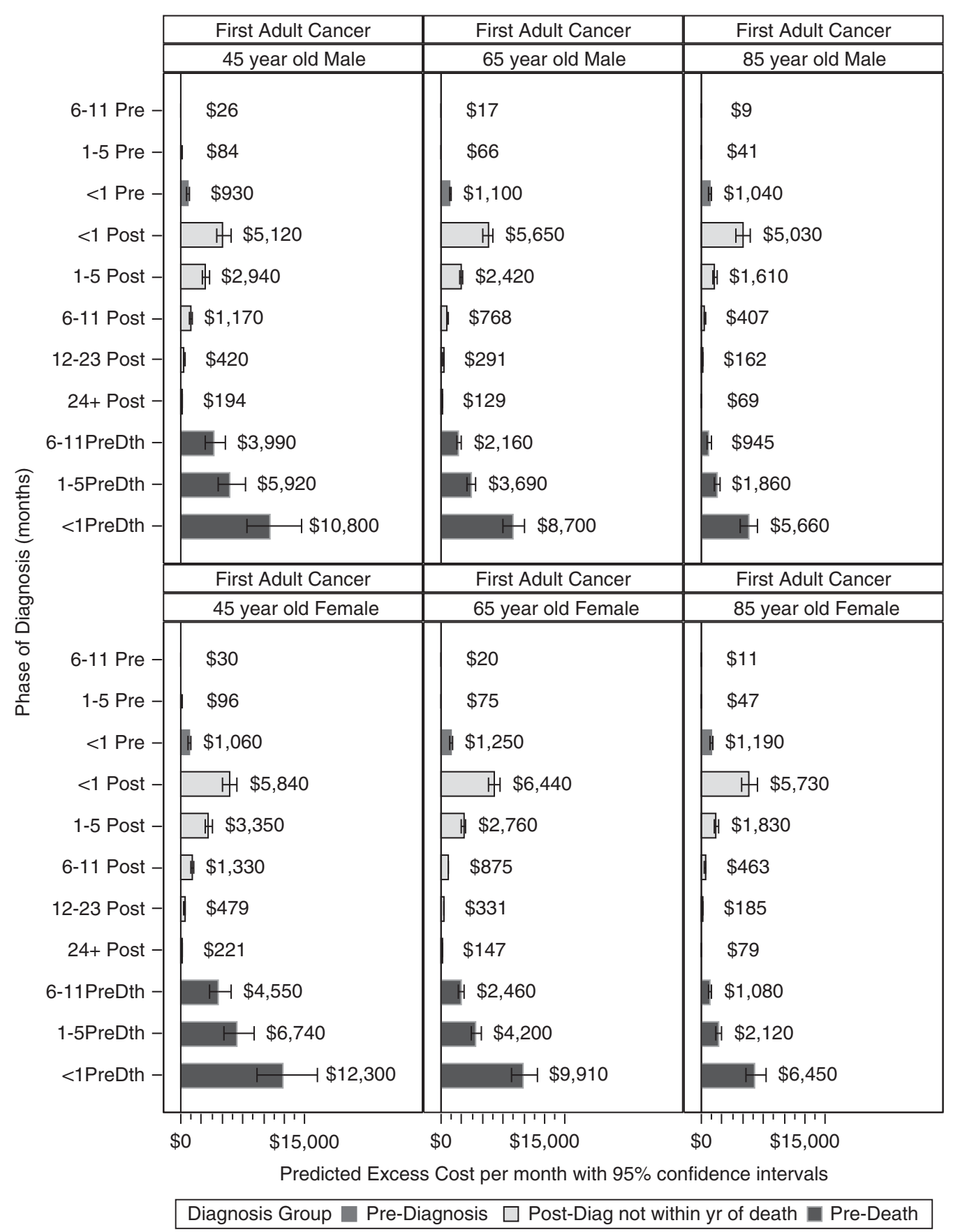

FIGURE 1. Excess health system cost per person per month of "first adult cancer" diagnosis by time preceding and postdiagnosis, separating off the 12 months before death from cancer, by sex and age. Error bars are $95 \%$ confidence intervals. Costs in NZ\$, inflation-adjusted to 2011, using regression modeling of 2006-2011 data to predict 2011 values.

still subject to limitations. Firstly, not all public expenditure that can be attributed to individuals is yet captured in HealthTracker: Vote:Health funding through disability support services, maternity and mental health care are not yet accessible to academic researchers; this is work in progress, and will probably see the total percentage of Vote:Health allocated rise from $50 \%$ to around $75 \%-80 \%$. [There will always be an unallocated proportion due to costs not attributed to individual events (eg, public health, training, administration)]. In addition, injury costs are not yet allocated. For these yet to be allocated costs to bias the results in this study, it would require these costs to be more (or less) likely to occur among cancer patients than an "average citizen." This is probably not the case for injury and maternity care. For disability support services and mental health they are probably more likely to occur among cancer patients, therefore we have probably underestimated some of 


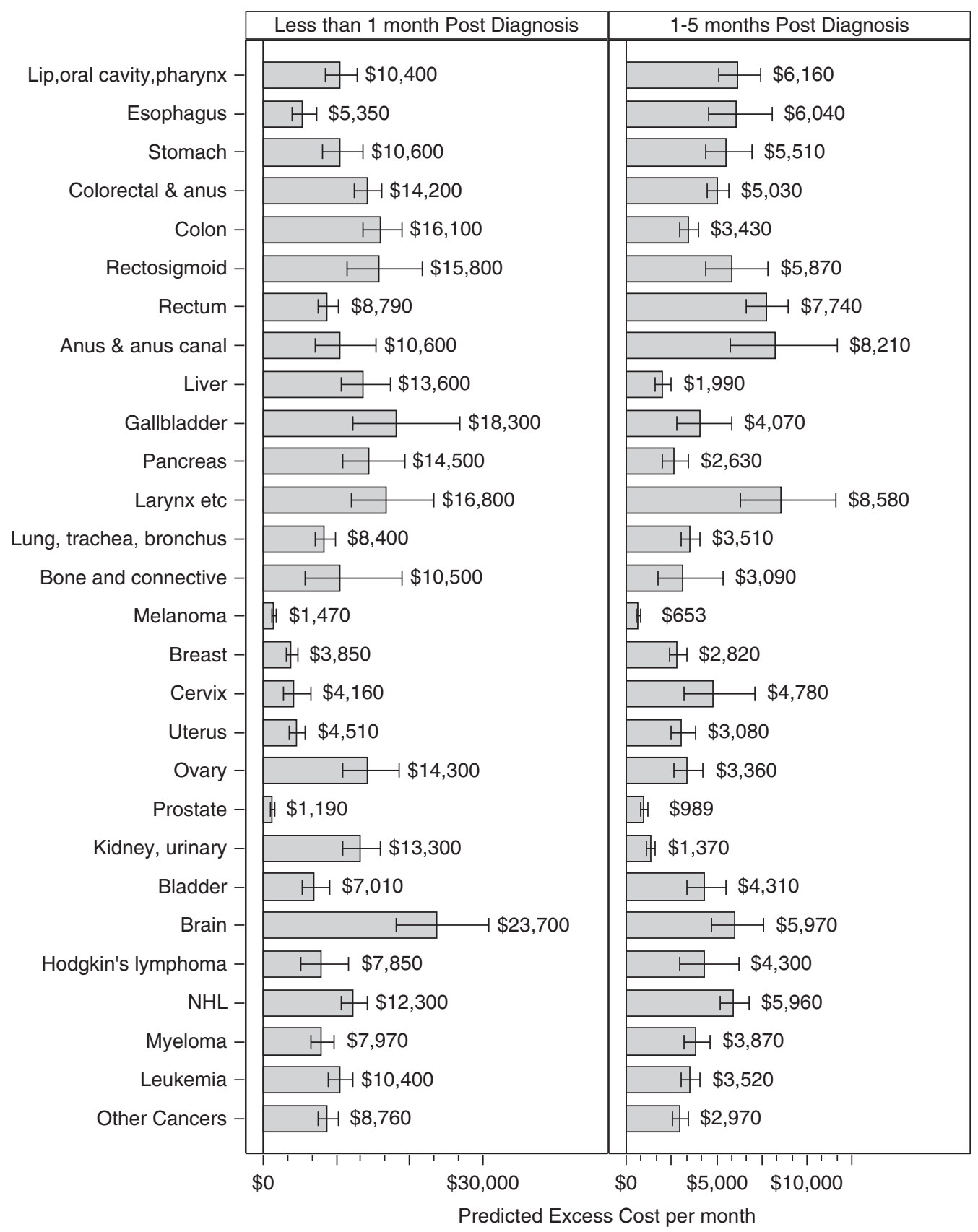

FIGURE 2. Excess health system costs per person per month for multiple cancers, compared across cancers for 65 -year-old males (or females if female-only cancer) for: first month postdiagnosis, and 1-5 months postdiagnosis. Error bars are $95 \%$ confidence intervals. Costs in NZ\$, inflation-adjusted to 2011.

the true excess publicly funded cancer costs. Put another way, our $6.5 \%$ estimate of all Vote:Health being due to cancer is probably an underestimate, and might be closer to $7 \%$ (eg, if $2 \%-3 \%$ of the yet to be allocated $25 \%$ of Vote:Health was due to cancer).

Secondly, private expenditure is not included in HealthTracker. If combined public and private expenditure is required, then estimates need to be increased. One solution is to simply scale up all estimates by $20 \%$, given that $17 \%$ of all health expenditure in New Zealand is estimated to be private $^{23}$ (ie, $20 \%=1 / 0.83-1$ ). One likely problem with this scaling for cancers is that some cancers (eg, lung) are more likely to be managed in public than other cancers (eg, prostate and breast). To our knowledge, there are limited data to make these cancer-specific adjustments; and so this would be useful additional research. Thirdly, immigration data were not linked in with these files - although this is unlikely to impact significantly on our estimates.

The results for specific cancers that comprise the largest fraction of cancer costs (eg, colorectal at $14.7 \%$ and 


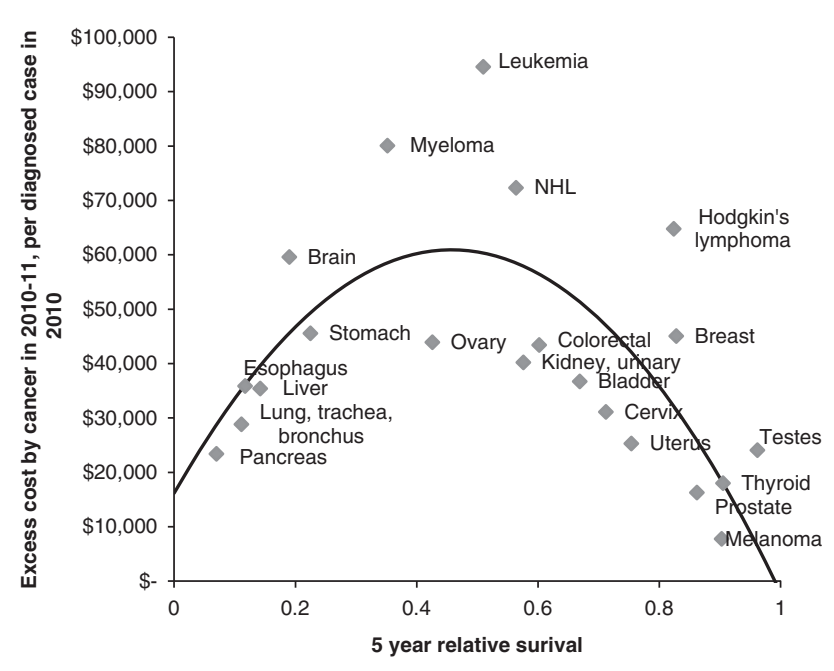

FIGURE 3. Association of annual excess costs by cancer per diagnosed case, with the 5-year relative survival (RS) ratio for that cancer type. Line is ordinary least squares regression line for equation: $\operatorname{cost}=16128+(195869 \times \mathrm{RS})-(214145 \times \mathrm{RS})$; where $P$ for RS and RS coefficients were 0.004 and 0.001 , respectively. RSR indicate relative survival risk.

breast at $14.4 \%$ ) might be of interest to health sector policy makers. These cancers are relatively common and frequently involve surgery and often other relatively expensive treatment modalities (eg, chemotherapy and radiotherapy). Improved investment in primary and secondary prevention may assist with their cost control, for example, addressing diet and alcohol risk factors for breast cancer ${ }^{24}$ and adopting screening programs for the secondary prevention of colorectal cancer. ${ }^{25}$ For many other cancer types (especially respiratory tract ones), improvements in tobacco control will also assist with both disease prevention and cost control.

In conclusion, the results of this study illustrate how cancer-specific excess costs can be estimated using a large and comprehensive administrative dataset. This approach is useful in deriving disease-specific costs that can be used in health economic models for other chronic disease areas. These costs should also be of relevance to economists, policy makers, and health service researchers, particularly those interested in forecasting future health care costs of people with various cancers, or wishing to model the cost-effectiveness of interventions aimed at preventing or treating these diseases.

\section{REFERENCES}

1. Murray CJL, Vos T, Lozano R, et al. Disability-adjusted life years (DALYs) for 291 diseases and injuries in 21 regions, 1990-2010: a systematic analysis for the Global Burden of Disease Study 2010. Lancet. 2012;380:2197-2223.
2. Siddiqui M, Rajkumar SV. The high cost of cancer drugs and what we can do about it. Mayo Clin Proc. 2012;87:935-943.

3. Smith TJ, Hillner BE. Bending the cost curve in cancer care. $N$ Eng $J$ Med. 2011;364:2060-2065.

4. Neumann PJ, Bliss SK, Chambers JD. Therapies for advanced cancers pose a special challenge for health technology assessment organizations in many countries. Health Aff. 2012;31:700-708.

5. Jönsson B. Technology assessment for new oncology drugs. Clin Cancer Res. 2013;19:6-11.

6. Van Baal PHM, Feenstra TL, Polder JJ, et al. Economic evaluation and the postponement of health care costs. Health Econ. 2011;20: $432-445$.

7. Wolstenholme J. Counting the Costs of Cancer Care: Breast, Cervical and Lung Cancer in Trent. Nottingham: University of Nottingham; 2001.

8. Mariotto AB, Yabroff KR, Shao Y, et al. Projections of the cost of cancer care in the United States: 2010-2020. J Natl Cancer Inst. 2011;103:117-128.

9. Ministry of Health. New Zealand Casemix Framework for Publicly Funded Hospitals, Including WIESNZ11 Methodology and Casemix Purchase Unit Allocation. Wellington: Ministry of Health; 2013.

10. Costilla R, Tobias M, Blakely T. The burden of cancer in New Zealand: a comparison of incidence and DALY metrics and its relevance for ethnic disparities. Aust N Z J Public Health. 2013;37:218-225.

11. Ministry of Health. Cancer: New Registrations and Deaths. Wellington: Ministry of Health; 2010.

12. Ministry of Health. Cancer Patient Survival Covering the Period 1994 to 2007. Wellington: Ministry of Health; 2010.

13. Brown ML, Riley GF, Schussler N, et al. Estimating health care costs related to cancer treatment from SEER-Medicare data. Med Care. 2002;40:IV-104-IV-117.

14. Fireman BH, Quesenberry CP, Somkin CP, et al. Cost of care for cancer in a health maintenance organization. Health Care Financ Rev. 1997; 18:51-76.

15. Lipscomb J, Barnett PG, Brown ML, et al. Advancing the science of health care costing. Med Care. 2009;47:S120-S126.

16. Barlow WE. Overview of methods to estimate the medical costs of cancer. Med Care. 2009;47:S33-S36.

17. Blakely T, Atkinson J, Kvizhinadze G, et al. Health system costs by sex, age and proximity to death, and implications for estimation of future expenditure. NZMJ. 2014;127:12-25.

18. Dickman PW, Sloggett A, Hills M, et al. Regression models for relative survival. Stat Med. 2004;23:51-64.

19. Sarfati D, Blakely T, Pearce N. Measuring cancer survival in populations: relative survival vs cancer-specific survival. Int $J$ Epidemiol. 2010;39:598-610

20. Yabroff KR, Warren JL, Brown ML. Costs of cancer care in the USA: a descriptive review. Nat Clin Pract Oncol. 2007;4:643-656.

21. Brown ML, Lipscomb J, Snyder C. The burden of illness of cancer: economic cost and quality of life. Annu Rev Public Health. 2001;22: $91-113$.

22. Ministry of Health. The Price of Cancer: The Public Price of Registered Cancer in New Zealand. Wellington: Ministry of Health; 2011.

23. New Zealand Treasury. Health Projections and Policy Options Background Paper for the 2013 Statement on the Long-Term Fiscal Position. Wellington: New Zealand Treasury; 2013.

24. Brennan SF, Cantwell MM, Cardwell CR, et al. Dietary patterns and breast cancer risk: a systematic review and meta-analysis. Am Clin Nutr. 2010;91:1294-1302.

25. Littlejohn C, Hilton S, Macfarlane GJ, et al. Systematic review and meta-analysis of the evidence for flexible sigmoidoscopy as a screening method for the prevention of colorectal cancer. Br J Surg. 2012;99: $1488-1500$. 